\title{
Bow-tie diodes for terahertz imaging: a comparative study
}

\author{
Rimvydas Venckevičius, ${ }^{1}$ Irmantas Kašalynas, ${ }^{1, *}$ and Gintaras Valušis ${ }^{1,2} \dagger$ \\ ${ }^{I}$ Optoelectronics Department, Semiconductor Physics Institute, Center for Physical Sciences and Technology, \\ A. Goštauto 11, LT-01108 Vilnius, Lithuania, \\ ${ }^{2}$ Semiconductor Physics Department, Faculty of Physics, Vilnius University, Sauletekio av. 9, Bldg. III, \\ LT-10222 Vilnius, Lithuania
}

Received August 21, 2012; accepted September 18, 2012; published September 30, 2012

\begin{abstract}
The concept of broadband bow-tie diodes as terahertz sensors and their possible application for imaging is presented. Different bow-tie sensors fabricated of planar GaAs, modulation doped GaAs/AlGaAs structures and InGaAs layers are compared and discussed. It is demonstrated experimentally that the sensitivity of all studied devices at room temperature is nearly independent of frequency below $1 \mathrm{THz}$ and amounts to $0.3 \mathrm{~V} / \mathrm{W}$ for GaAs-based structures and $10 \mathrm{~V} / \mathrm{W}$ for InGaAs sensors. Room temperature terahertz images are recorded at $0.71 \mathrm{THz}$ and $1.4 \mathrm{THz}$. Their parameters are considered in comparison with images taken by using commercially available pyroelectric sensors.
\end{abstract}

The implementation of terahertz $(\mathrm{THz})$ radiation in security applications and materials inspection requires reliable and compact solutions in the development of room temperature imaging systems. In these kinds of requirements, preference goes to solid-state-based solutions. A recent major breakthrough made in all-solidstate tunable sources has opened a promising route in the progress of room temperature $\mathrm{THz}$ emitters [1]. As concerns room temperature solid-state $\mathrm{THz}$ detectors, one can mention Schottky diodes [2], nanometric field effect transistors [3, 4] and microbolometers [5, 6]. Very recent achievements in compact room temperature spectroscopic [7] and heterodyne $\mathrm{THz}$ imaging [8] allowed one to extend the family of compact $\mathrm{THz}$ sensors by adding a member of so-called InGaAs based bow-tie (BT) diodes. The sensors exhibit broadband operation as well as relatively simple device production and good reliability.

In this paper, we present a comparative study of different BT diode sensors. An operational principle and spectral features of the device is discussed, BT diode's parameters based on InGaAs layers, modulation doped GaAs/AlGaAs structures and planar GaAs are compared. Terahertz images recorded at room temperature using InGaAs BTs are compared with images taken by using commercially available pyro-electric sensors; feasibility for real-time $\mathrm{THz}$ imaging is considered, too.

The bow-tie diode uses an idea to combine the performance of a bow-tie antenna as a coupler of incident

\footnotetext{
*E-mail: irmantak@ktl.mii.lt,

† E-mail: valusis@pfi.lt
}

radiation and the semiconducting part as radiation sensing media. The diode displays structurally broken symmetry as depicted in Fig. 1: One of the two semiconductor leaves is metalized in order to couple incident radiation into the second one, where high mobility electrons are heated nonuniformly by incident $\mathrm{THz}$ radiation [9]. As a result, the voltage signal across the leaves is induced without any application of a bias voltage.

Three different types of bow-tie diodes having the same geometry, but fabricated of different materials, were studied. The first one was GaAs/AlGaAs modulation doped structures consisting of a 20-nm-thick GaAs cap layer, Si-doped $\left(1 \times 10^{18} \mathrm{~cm}^{-3}\right) 80 \mathrm{~nm} \mathrm{Al}_{0.25} \mathrm{Ga}_{0.75} \mathrm{As}$ layer, an undoped $\mathrm{Al}_{0.25} \mathrm{Ga}_{0.75} \mathrm{As}$ spacer of $45 \mathrm{~nm}$, and $1 \mu \mathrm{m}$ of undoped GaAs. The sheet electron density and the mobility at room temperature are $5.5 \times 10^{11} \mathrm{~cm}^{-2}$ and $4700 \mathrm{~cm}^{2} / \mathrm{V} \cdot \mathrm{s}$, respectively. (Details can be found in [9]).

The active part of the InGaAs-based device is an $\mathrm{In}_{0.54} \mathrm{Ga}_{0.46} \mathrm{As}$ layer of $534 \mathrm{~nm}$ thickness grown on InP (001) substrate with one InAs monolayer in between. The electron concentration and mobility at room temperature are about $2 \times 10^{15} \mathrm{~cm}^{-3}$ and $13300 \mathrm{~cm}^{2} / \mathrm{V} \cdot \mathrm{s}$, respectively. (Details can be found in [10]).

The third type of diode was a planar GaAs structure manufactured on the base of $n-n^{+}-\mathrm{GaAs}$ epitaxial layer. The thickness of the diode was $3 \mu \mathrm{m}, n$-part doping was $1 \times 10^{15} \mathrm{~cm}^{-3}$, while that of $n^{+}$-part was about $2 \times 10^{18} \mathrm{~cm}^{-3}$. An elastic polyimide film of $10 \mu \mathrm{m}$ thickness served as a mechanical support for the diode. (Details can be found in [11]).

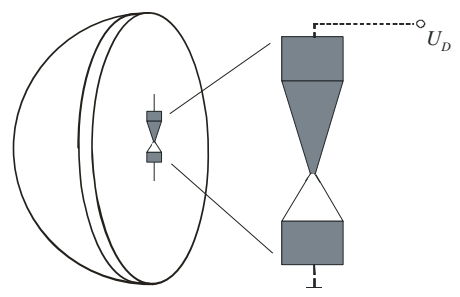

Fig. 1. Left panel - bow-tie-diode coupled to a silicon lens. Right panel - shape of the diode. Grey-coloured areas denote metalized parts, white area shows semiconducting part containing high mobility electrons. 


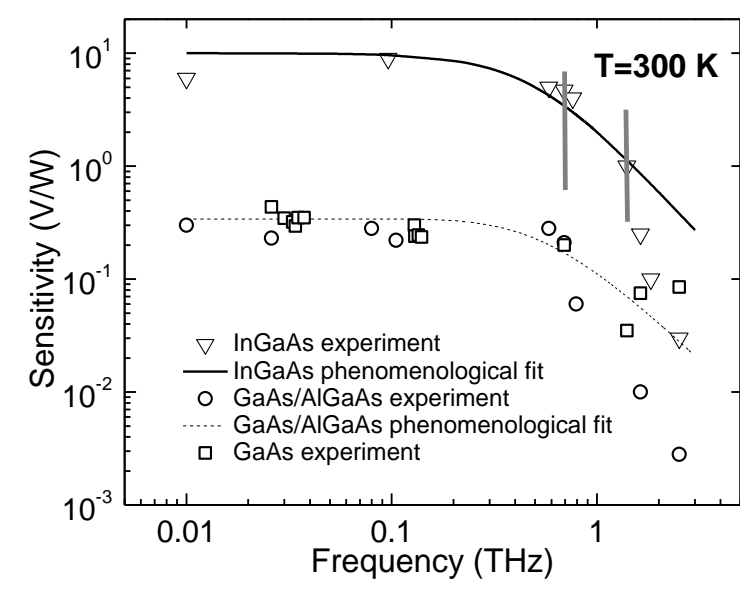

Fig. 2. Frequency dependence of voltage sensitivity of an InGaAsbased device, GaAs/AlGaAs modulation doped-based bow tie diode (taken from [9]) and planar $n-n^{+}-\mathrm{GaAs}$ structure-based device (taken from [11]) with apex size of $12 \mu \mathrm{m}$. Points denote experimental data, lines - the phenomenological fit. Grey bars in InGaAs diode data plot label frequencies of the recorded images given in Fig. 4.

To process the devices we have used conventional optical lithography and wet etching. The details of processing technology and contacts fabrication procedures for different devices are described in Ref. [9]-[11].

Three different types of high-frequency sources were employed in the experiments. Magnetrons operating in a pulsed mode of $2-20 \mu$ s duration and a repetition rate of $40 \mathrm{~Hz}$ were used at $10 \mathrm{GHz}$ and $35 \mathrm{GHz}$, backward wave oscillators served as a source within the range of $78-118 \mathrm{GHz}$, while an optically pumped molecular laser operating in a continuous wave regime was used as an emitter in the $\mathrm{THz}$ range - from $0.584 \mathrm{THz}$ up to $2.52 \mathrm{THz}$.

In the latter experiments, the signal was measured by a lock-in amplifier with a chopper frequency of $67 \mathrm{~Hz}$; in frequencies below $118 \mathrm{GHz}$ the signal was recorded by an oscilloscope.

Frequency dependences of the voltage sensitivity of all studied devices are shown in Fig. 2. As seen, the sensitivity of all studied devices displays an inherent feature of weak dependence on frequency from $10 \mathrm{GHz}$ up to $1 \mathrm{THz}$. It is seen that the sensitivity of the InGaAs-based diodes is more than an order of magnitude higher (close to $10 \mathrm{~V} / \mathrm{W}$ ) in comparison to both GaAs-based structures, which is close to $0.3 \mathrm{~V} / \mathrm{W}$. It is reasonable due to much higher electron mobility in InGaAs than in GaAs at room temperature and better coupling. It is evident that the experimental points below $1 \mathrm{THz}$ are well fitted by phenomenological approach [10]. With a further increase of frequency the sensitivity drops down faster than phenomenological estimates predicted due to weaker coupling of radiation as was evidenced via finitedifference time domain calculations [9]. Experimentally observed peculiarity in a planar $n-n^{+}$-GaAs structure - an increase of sensitivity at $1.63 \mathrm{THz}$ and $2.52 \mathrm{THz}$ instead of a general decreasing trend - can be explained by antenna resonances which can be pronounced in such an antennalike structure [12].

Therefore, the InGaAs-based diodes due to their sensitivity can be applied for imaging aims. First images taken using InGaAs bow-tie diodes in the subTHz range, at $0.693 \mathrm{THz}$, were demonstrated showing the features of a plastic capsule half-filled with pharmaceutical powder [13]. Here we focused our attention on compact spectroscopic imaging as the device demonstrates operation over a broadband frequency band, up to 2.5THz. Moreover, we compared imaging parameters using BTs diodes and commercially available pyroelectric sensors ( $\varnothing 9 \mathrm{~mm}$ diameter Molectron P1-19).

To demonstrate $\mathrm{THz}$ imaging we chose InGaAs bow tie diodes coupled with silicon lenses.

For spectroscopic imaging, two samples of polytetrafluoroethylene (PTFE) - one with explosive simulator powder inside the PTFE matrix, and the other, pure PTFE, were prepared. The latter served as a reference. The samples were packed in a box opaque for visible light, but transparent for $\mathrm{THz}$ radiation. The photo of the box enclosure and its contents is given in Fig. 3 .

Two laser emission lines of an optically pumped molecular laser - one far from spectral peculiarities of an explosive simulator, at $0.716 \mathrm{THz}$, and the second - rather close to the explosive simulator transmission minimum, at $1.4 \mathrm{THz}$ - were used to record images, as it was described in Ref. [7]. The results are shown in Fig. 4. It is clearly seen that the explosive simulator containing the tablet exhibits differently "shadowed" images due to stronger absorbance at each frequency. In contrast, the "color" of the pure PTFE reference sample practically shows no changes since this sample has no spectral peculiarities in its spectra $[7,14]$.

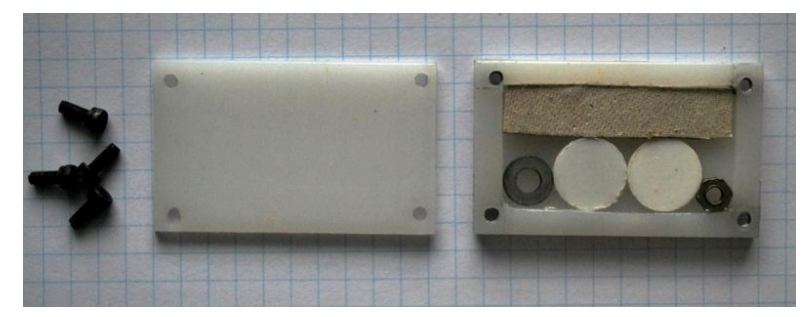

Fig. 3. Left panel - photo of a box closure for imaging demonstration of packaged objects. The box made of high density polyethylene (HDPE) is opaque for visible light, but transparent for $\mathrm{THz}$ radiation. Right panel - photo of objects under test. Metallic washers served as references, a piece of carton was used to fix the white colour PTFE tablets. The left one is pure PTFE, the right one is PTFE with an explosive simulator. 


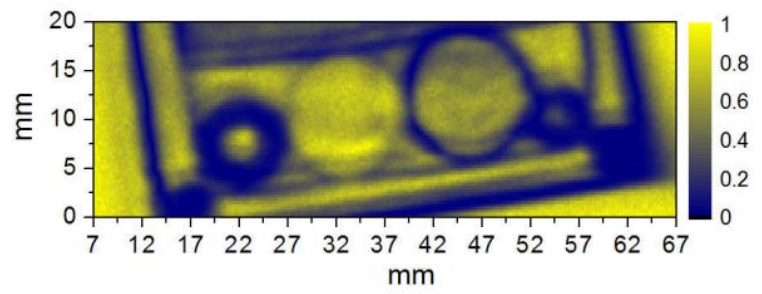

Fig. 4a. Terahertz image recorded using an InGaAs diode at $0.76 \mathrm{THz}$. The laser power was about $2 \mathrm{~mW}$, lock-in time constant is $10 \mathrm{~ms}$, modulation frequency is $500 \mathrm{~Hz}$, signal-to-noise ratio is $23 \mathrm{~dB}$,

image recording time $-11 \mathrm{~min}, 67 \times 1379$ pixels, pixel size $-300 \mu \mathrm{m}$.

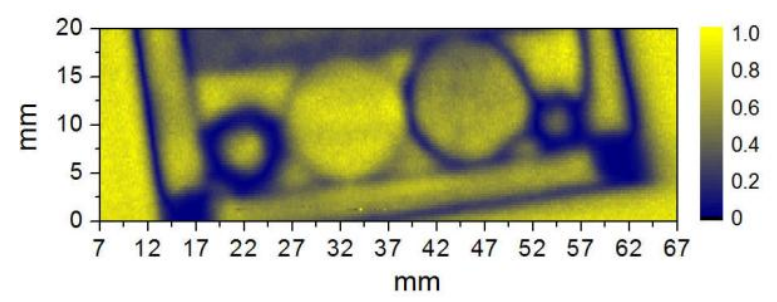

Fig. 4b. Terahertz image recorded at $0.76 \mathrm{THz}$ using $\varnothing 9 \mathrm{~mm}$ diameter pyro-electric sensor. Signal-to-noise ratio is $21 \mathrm{~dB}$. All other parameters are the same as is stated in Fig. 4a.

The comparison of images of metallic washers at different frequencies illustrates an increase in spatial resolution at higher $\mathrm{THz}$ frequencies.

If one compares the quality of images taken by using InGaAs bow-tie diodes with pyro-electric sensors, one can see that the devices are rather comparable for imaging aims, the values of signal-to-noise ratios in both are rather close to each other $-23 \mathrm{~dB}$ and $21 \mathrm{~dB}$ at $0.76 \mathrm{THz}$ and $25 \mathrm{~dB}$ and $30 \mathrm{~dB}$ at $1.4 \mathrm{THz}$, respectively.

For real-time imaging aims, the key-issue is the operation speed of the $\mathrm{THz}$ sensor. It was measured that the response time of the BT diode is less than few nanoseconds [13]. Moreover, it was demonstrated that an InGaAs-based bow-tie diode is well suited for fast spectroscopic $\mathrm{THz}$ imaging. Measurements performed with the BT diodes at $2.52 \mathrm{THz}$ frequency have shown that, without losing any $\mathrm{THz}$ image quality, images can be recorded via increasing the $\mathrm{THz}$ beam modulation frequency and the scanning speed up to $30 \mathrm{kHz}$ and $300 \mathrm{~mm} / \mathrm{s}$, respectively [7]. If the bow-tie diode is replaced by a bandwidth limited commercial pyroelectric detector, it results in a smoothed profile leading to an image blurring effect and incorrect transmittance values.

An important element of imaging quality is to compare noise properties of different devices via noiseequivalent-power (NEP). Bow-tie NEP was estimated to be of $4 \mathrm{nW} / \sqrt{\mathrm{Hz}}$ [8], while that for silicon CMOS field effect transistors (FETs) - $10 \mathrm{pW} / \sqrt{\mathrm{Hz}}$ [15] and $43 \mathrm{pW} / \sqrt{\mathrm{Hz}}$ [16]. The reported NEP value for commercial nanometric GaAs FETs transistors is about $10 \mathrm{nW} / \sqrt{\mathrm{Hz}}[17]$.

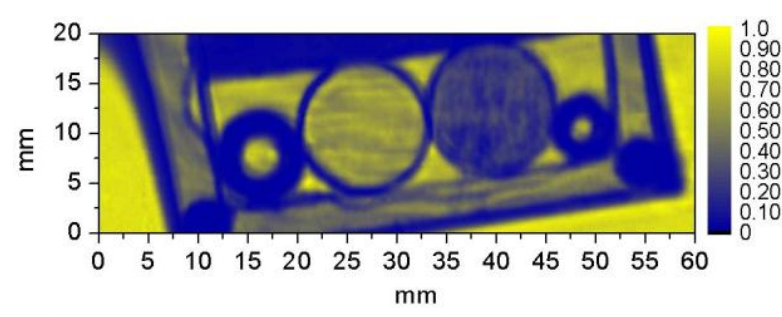

Fig. 4c. Terahertz image recorded using InGaAs diode at $1.4 \mathrm{THz}$. The laser power was set to $10 \mathrm{~mW}$, lock-in time constant is $10 \mathrm{~ms}$, modulation frequency is $1 \mathrm{kHz}$, signal-to-noise ratio is $25 \mathrm{~dB}$, image recording time $-11 \mathrm{~min}$, Pixels $67 \times 1379$, pixel size is $200 \mu \mathrm{m}$.

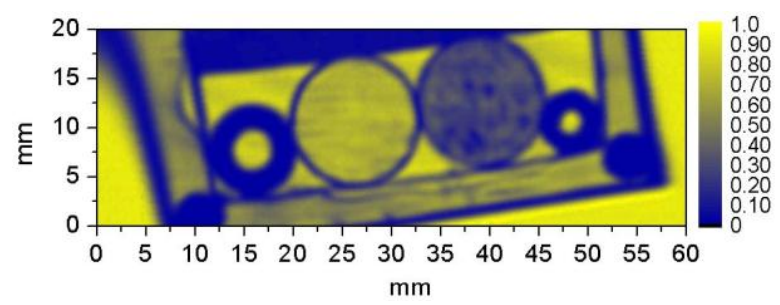

Fig. 4d. Terahertz image recorded at $1.4 \mathrm{THz}$ using $\varnothing 5 \mathrm{~mm}$ diameter pyro-electric sensor. Signal-to-noise ratio is $30 \mathrm{~dB}$. All other parameters are the same as is stated in Fig. 4c.

The most impressive number is reported for $\mathrm{THz}$ microbolometers - NEP is found to be $1 \mathrm{pW} / \sqrt{\mathrm{Hz}}$ [18].

In summary, the InGaAs-based bow-tie diode was found to be well-suitable for fast $\mathrm{THz}$ imaging at room temperature. The parameters of different bow-tie sensors were discussed and applications for spectroscopic and real-time $\mathrm{THz}$ imaging were considered comparing the parameters of bow-tie diodes with relevant values of pyroelectric sensors, field effect transistors and microbolometers.

The work was supported by the Research Council of Lithuania under contract HeTeFo MIP-093/2012.

\section{References}

[1] A. Maestrini et al., IEEE Trans. Terahertz Sci. Technol. 2, 177 (2012).

[2] P.H. Siegel, IEEE Trans. Microwave Theory Tech. 50, 910 (2002).

[3] W. Knap et al., Appl. Phys. Lett. 80, 3433 (2002).

[4] A. Lisauskas et al., Electron. Lett. 44, 408 (2008).

[5] A.W.M. Lee et al., IEEE Photon. Technol. Lett. 18, 1415 (2006).

[6] Duy-Thong Nguyen et al., IEEE Trans. Terahertz Sci. Technol. 2, 299 (2012).

[7] I. Kašalynas et al., J. Appl. Phys. 110, 114505 (2011).

[8] L. Minkevičius et al., Appl. Phys. Lett. 99, 131101 (2011).

[9] D. Seliuta et al., Electron. Lett. 40, 631 (2004).

[10] D. Seliuta et al., Electron. Lett. 42, 825 (2006).

[11] A. Sužiedèlis et al., J. Appl. Phys. 93, 3034 (2003).

[12] L. Minkevičius et al., Lith. J. Phys. 50, 173 (2010)

[13] I. Kašalynas et al., Electron Lett. 45, 833 (2009).

[14] L. Minkevičius et al., Proc. 19th Intern. Conf. Microwaves, Radar, and Wireless Comm. MIKON 2012, IEEE 2012, p.105.

[15] F. Schuster et al, Opt. Express 19, 7827 (2011).

[16] S. Boppel at al., Electron. Lett. 47, 661 (2011).

[17] S. Nadar et al., J. Appl. Phys. 108, 054508 (2010).

[18] Duy-Thong Nguyen et al., Proc. 19th Intern. Conf. Microwaves, Radar, and Wireless Comm. MIKON 2012, IEEE 2012, p.116. 